Korean CAREX, we focused on estimating the exposure intensity of lead across industries, which is a suspected carcinogen. Methods We extracted airborne lead measurements from the work environment measurement database (WEMD) which is the Korean nationwide measurement database. In addition, we elicited the experts' opinion about lead exposure intensity across industries by conducting a questionnaire. Experts provided estimates of lead exposure levels as the boundary of the 5 th and 95th percentiles. We assumed that experts provided their estimates based on the assumption of log-normal distributions of exposure. First, for each industry, estimates of logtransformed geometric means (logGM) and log-transformed geometric standard deviations (logGSD) were extracted from the experts' responses, followed by combining them to quantify the experts' prior Normal-Inverse-Gamma prior distribution. Then, the corresponding $\log G M$ and $\log$ GSD from lead measurement data for each industry were updated with the experts' prior distribution through a Bayesian framework, yielding posterior distributions of $\log \mathrm{GM}$ and $\log \mathrm{GSD}$.

Results WEMD contains 83035 airborne lead measurements collected between 2002-2007. Total 17 occupational hygiene professionals with more than 20 year experience provided lead exposure estimates. In industries where measurement data is abundant, the measurement data dominate the posterior exposure estimates, while in industries with a limited number of measurements, experts' opinion played an important role in determining posterior exposure estimates. For example, rubber manufacturing industry with 246 measurements (GM 1.72; GSD 1.94) and 6 experts' responses (GM 0.79; GSD 6.73) showed posterior exposure estimates of GM 1.60 and GSD 2.34 .

Conclusions Our method of estimating the exposure intensity of CAREX may introduce an unbiased approach to the development process by utilising both prior knowledge of experts and measurement data simultaneously. In addition, it will supply a framework for future updates.

\section{ESTIMATION OF LEAD EXPOSURE PREVALENCE IN KOREAN POPULATION THROUGH COMBINING MULTIPLE EXPERTS' JUDGMENT BASED ON OBJECTIVE DATA SOURCES}

${ }^{1}$ Dong-Hee Koh*, ${ }^{2}$ Sang-Gil Lee, ${ }^{3}$ Hwan-Cheol Kim, ${ }^{4}$ Ju-Hyun Park, ${ }^{5}$ Sangiun Choi, ${ }^{1}$ Hyejung Jung, ${ }^{6}$ Dong-Uk Park. 'Department of Occupational and Environmental Medicine, International St. Mary's Hospital, Catholic Kwandong University, Incheon, Korea; ${ }^{2}$ Occupational Safety and Health Research Institute, Korea Occupational Safety and Health Agency, Ulsan, Korea; ${ }^{3}$ Department of Occupational and Environmental Medicine, Inha University, Incheon, Korea; ${ }^{4}$ Department of Statistics, Dongguk University, Seoul, Korea; ${ }^{5}$ Department of Occupational Health, Catholic University of Daegu, Daegu, Korea; ${ }^{6}$ Department of Environmental Health, Korea National Open University, Seoul, Korea

\subsection{6/oemed-2018-ICOHabstracts.343}

Objectives Estimation of exposure prevalences of carcinogens is important for preventing occupational cancers. For develop-

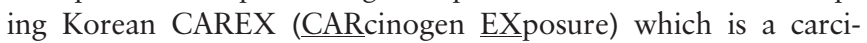
nogen surveillance system employed in many countries, as an exemplary carcinogen, we estimated lead exposure prevalences in Korean working population.

Methods We used three nationwide data sources to retrieve objective default estimates of lead exposure prevalence across industries: the work-environment monitoring database (WEMD), the special health examination database (SHED), and the Work Environment Condition Survey (WECS).
Furthermore, we surveyed experts for their judgment about lead exposure prevalence across industries after reviewing the default estimates computed from aforementioned three exposure databases. We developed various estimation methods combining experts' judgment, and then compared with each other. The 2010 Census was used as the reference population to estimate the number of lead-exposed workers for the 228 industries through multiplying the exposure prevalence with the number of workers in each industry.

Results Default estimates of exposure prevalence for 228 industries were calculated using the WEMD and SHED collected between 2009-2011, and the 2009 WECS. A total of 52 experts who have 20 or more experience in industrial hygiene practice participated in the study and provided their judgment on the lead exposure prevalences as a number for the 228 industries. Among various estimation methods, the median values of experts' responses were selected for our estimates of lead exposure prevalence in each industry. As a result, a total of 129,247 Korean workers was estimated to be exposed to lead in the year of 2010.

Conclusions We developed a method estimating exposure prevalences combining experts' judgment based on objective databases. Our effort for estimating the exposure prevalences of CAREX may introduce an unbiased approach to the development process accounting for uncertainty of exposure.

\section{BIG DATA AND OCCUPATIONAL HEALTH SURVEILLANCE: USE OF FRENCH MEDICO- ADMINISTRATIVE DATABASES FOR HYPOTHESIS GENERATION REGARDING OCCUPATIONAL RISKS IN AGRICULTURE}

${ }^{1,2} \mathrm{C}$ Maugard ${ }^{*},{ }^{1} \mathrm{D}$ Bosson-Rieutort, ${ }^{2} \mathrm{O}$ François, ${ }^{1,3} \mathrm{~V}$ Bonneterre. ${ }^{1}$ EPSP Team, TIMC-IMAG Laboratory, Grenoble-Alpes University, Grenoble, France; ${ }^{2} B C M$ Team, TIMC-IMAG Laboratory, Grenoble-Alpes University, Grenoble, France; ${ }^{3}$ Occupational Medicine and Health Dpt., CHU Grenoble-Alpes, Grenoble, France

\subsection{6/oemed-2018-ICOHabstracts.344}

Introduction Surveillance of diseases and associated exposures is a major issue in occupational health, especially for identifying new work-related diseases. In addition to classical epidemiology (hypothesis-driven studies), complementary methods relying on data mining of health insurance data must be developed for early detection of work-related diseases, without prior hypothesis.

Methods Data from the insurance fund of French agricultural workers ('Mutualité Sociale Agricole', MSA), which covers about 3 million individuals, were considered. The study population included all self-employed or employee affiliates from the 2006-2015 period. MSA holds medico-administrative databases, which include information on occupational activities as well as long-term diseases identified with ICD-10 codes. Following authorisation of MSA and of the French National Commission on Informatics and Liberty, these databases were cross-linked for the first time. After preliminary data treatments, generalised linear models and latent factor models were applied to detect over-represented statistical associations between occupational activity and long-term disease. Results were represented as p-value plots in order to highlight the key statistical signals.

Results The population covered by this study accounted for more than 2 million individuals $(n=2,250,177)$ with a majority of men (64\%) and an average age of 46 years. Within this 